\title{
Homo sapiens: Wie viel Prozent Mensch?
}

\author{
Von Körperflora und „Krankenhauskeimen“
}

\section{Ines Kappstein}

Der Mensch ist „eingetaucht“ in Bakterien und andere Mikroorganismen: Sie leben in unserem Körper und sind in unserer Nahrung und allem, was uns umgibt, immer vorhanden. Wir können ohne sie nicht leben.

Betrachten wir den Menschen und seine natürliche Mikroflora rechnerisch, dann stellt sich heraus, dass der mikrobielle Anteil größer ist als der menschliche [1]: So macht die Mikroflora des Menschen mit ca. 1 - 2,5 kg lebender Bakterien zwar nur einen Prozentsatz von etwa 1 -3,5\% am Gesamtgewicht (bei $70 \mathrm{~kg}$ Körpergewicht) aus, beim Vergleich der Zellzahlen menschlicher und bakterieller Zellen dominieren die Bakterien bereits mit 90\%, aber bei der Anzahl der Gene schlagen die Bakterien den Menschen beispiellos mit einem Anteil von 99\%, d.h. „genetisch“ sind wir nur zu $1 \%$ menschlich.

Ohne Bakterien können wir deshalb nicht leben, weil sie eine bedeutende Rolle bei vielen Körperfunktionen spielen: Sie schützen uns beispielsweise vor Infektionen durch Erreger, die von außen an uns herantreten (wollen), indem sie solche Eindringlinge dadurch in Schach halten, dass sie sie durch ihre Überzahl und ihr gut austariertes Verhältnis zwischen Aerobiern und vor allem Anaerobiern an der Besiedlung des Körpers hindern. Ferner sind Bakterien maßgeblich an unserer normalen Verdauung beteiligt.

Die bakterielle Besiedlung des Menschen beginnt sofort nach der Geburt. „Spender“ sind dabei in erster Linie die Menschen in der Umgebung des Neugeborenen, allen voran natürlich die Mutter, aber auch jeder andere, der mit dem Kind insbesondere in direktem Kontakt steht. Somit werden Kinder, die in ihrer ersten Lebenszeit auf Intensivpflege angewiesen sind, zwangsläufig in der „unnatürlichen“ Umgebung ihrer Intensivstation primär bakteriell besiedelt. Auch wenn heute die Eltern auf neonatologischen Intensivstationen häufig auch direkten Haut- bzw. Körperkontakt mit ihren Kindern haben können (z. B. sogenanntes Kangarooing), hat das medizinische Personal doch den Hauptkontakt mit den Neugeborenen. Die Kinder erwerben somit einen wesentlichen Teil ihrer Körper- flora über das medizinische Personal [2]. Dazu gehören vor allem die Bakterien der normalen, „residenten“ Hautflora des Personals, wie insbesondere Koagulasenegative Staphylokokken, die, auch bei optimaler Versorgung, durch Händedesinfektion und andere Präventionsmaßnahmen nicht aus der unmittelbaren Umgebung des Neugeborenen zu eliminieren sind.

Die physiologische (oder kommensale) Mikroflora des Menschen ist ein bedeutendes Erregerreservoir für Infektionen und stellt einen schier unendlichen Pool für die Selektion resistenter Bakterien dar. Selektion, das ist quasi eine Filterung von Bakterien, die unter dem Einfluss von Antibiotika wegen mangelnder Empfindlichkeit (oder sogar Resistenz) schlicht übrig bleiben, weil die anderen - häufig gegen Antibiotika hoch empfindlichen Vertreter der normalen Körperflora der Wirksamkeit der Antibiotika zum Opfer fallen. Erst diese Störung des physiologischen mikrobiologischen Ökosystems macht es den weniger empfindlichen oder resistenten Bakterien möglich, sich wirksam anzusiedeln, also in einem Maße zu vermehren, dass sie vielleicht nicht unbedingt dominieren, aber doch zahlenmäßig so angewachsen sind, dass sie nun „mitspielen“ können: Clostridium difficile als häufige Ursache der Antibiotika-assoziierten Diarrhö oder diverse Bakterien der normalen Körperflora als potentielle Erreger von Infektionen im Zusammenhang mit den vielfältigen invasiven Maßnahmen, die die moderne Medizin heute vorhält. Gerade diese invasiven Maßnahmen sind es aber, die sogenannte Krankenhausinfektionen oft überhaupt erst möglich machen, weil sie potenziellen Infektionserregern (ob antibiotikaempfindlich oder -resistent) einen Zugang zu Körperregionen verschaffen, den diese normalerweise nicht haben: insbesondere Blasenkatheterisierung und Harnwegsinfektionen, intravasale Katheter und Bakteriämie, Beatmung und Pneumonie, Operationen und postoperative Infektionen im Operationsgebiet.

Das alles sind „Krankenhausinfektionen“. Sind aber die Erreger dieser Infektionen wirklich zutreffend als „Krankenhauskeime“ zu bezeichnen? Sind sie nicht, jedenfalls dann nicht, wenn man den Kontext betrach- 
tet, in dem sie in der Regel erst zu den Erregern sogenannter Krankenhausinfektionen werden. Meist ist es ist nicht das Krankenhaus, wo diese Erreger (ob resistent oder nicht) herkommen oder entstehen, sondern es ist der Mensch, d.h. der betroffene Patient, der selbst die Quelle seiner Infektionserreger ist.

Unbestritten werden Erreger von Krankenhausinfektionen auch von Patient zu Patient oder auf indirekterem Weg aus der von einem Patienten kontaminierten Umgebung durch mangelnde Beachtung der erforderlichen Präventionsmaßnahmen auf andere Patienten übertragen. Dies betrifft empfindliche und resistente Bakterien. Diese exogene Übertragung ist jedoch weniger häufig für „Krankenhausinfektionen“ verantwortlich, als gemeinhin angenommen, weil die meisten Infektionen im Zusammenhang mit invasiven medizinischen Maßnahmen aus der körpereigenen Flora des Patienten selbst stammen. Die sogenannten „Krankenhauskeime“ sind also keine Infektionserreger, die im Gebäude des Krankenhauses (in Kachelfugen oder auf Fußböden) darauf lauern, endlich „unschuldige“ Patienten befallen zu können. Vielmehr sind es in erster Linie die Erreger, die die betroffenen Patienten selbst beherbergen. Mitpatienten, das medizinische Personal und die sogenannte „unbelebte“ Umgebung sind dagegen weitaus seltener die Quelle nosokomialer Infektionen. Das bedeutet, dass „Krankenhauskeime“ in der Regel menschlichen Ursprungs sind, aber nicht primär aus der unbelebten Umgebung im Krankenhaus stammen. Deshalb gibt es auch den gelegentlich gerne angeführten sogenannten „Hauskeim“, z. B. als MRSA, nicht. Das zeigen molekularbiologische Untersuchungen, wonach nämlich in einer Klinik MRSA keineswegs gleich MRSA ist, sondern genotypisch eine Vielzahl von MRSA anzutreffen sind, die über die Zeit in ihrer Häufigkeit stark variieren können [3].

Wir haben es heute insbesondere bei der stationären Patientenversorgung nicht nur mit MRSA, sondern mit einer Vielzahl anderer (teilweise ebenfalls resistenter) Infektionserreger (C. difficile, Breitspektrum-Betalaktamase-[ESBL-]Bildner, Vancomycin-resistente Enterokokken, Noroviren) zu tun. Deshalb brauchen wir ein Konzept, das die normalen Übertragungswege aller dieser Erreger berücksichtigt, aber keine „Rezepte“, wie man sich bei diesem oder jenem Erreger "richtig“ verhält [4]. Denn die weitaus meisten Erreger von Krankenhausinfektionen werden durch Kontakt übertragen [5]. Davor schützen die Maßnahmen der sogenannte Standardhygiene. Damit sind Standardmaßnahmen in Standardsituationen gemeint, beispielsweise:
- Händedesinfektion und ggf. (Schutz-)Handschuhe (die anschließend stets wieder abgelegt werden müssen) vor dem Wundverbandswechsel und No-Touch-Technik währenddessen,

- Gebrauch von Handschuhen und Tragen von Schutzkleidung (Plastikschürze) für die Versorgung von Patienten mit (Gastro-)Enteritis,

- keine Hand-Gesichtskontakte bei der Patientenversorgung, bevor die ggf. verwendeten Handschuhe ausgezogen und/oder bevor die Hände desinfiziert wurden, als Schutz vor Selbstinokulation,

- Händehygiene (Händedesinfektion und ggf. Handschuhe) bei Versorgung von Patienten mit respiratorischen Infektionen und ggf. OP-Maske bei engem Kontakt (face-to-face) zum Schutz des Personals vor Tröpfchenkontakt mit respiratorischem Sekret sowie umgekehrt zum Schutz der Patienten bei entsprechender Erkrankung des Personals.

Dafür muss man nicht wissen, welcher Erreger, ggf. mit welcher Antibiotikaempfindlichkeit, in einer Wunde ist, oder ob Noroviren oder andere Erreger die Ursache einer Gastroenteritis sind, sondern man muss sich nach der klinischen Symptomatik richten, um entscheiden zu können, welche Barrieremaßnahmen vor einer direkten oder indirekten Erregerübertragung schützen.

\section{Literatur}

1 Wexler HM. Bacteroides: the good, the bad and the nitty-gritty. Clin Microbiol Rev 2007; 20: 593-621

2 Kappstein I. Nosokomiale Infektionen - Prävention, Antimikrobielle Therapie, Labor-Diagnostik.

4. Auflage. Stuttgart: Thieme, (in Vorbereitung; Erscheinungsdatum voraussichtlich Mai 2009)

3 Kappstein I, van der Mühlen K, Meschzan D et al. Prävention von MRSA-Übertragungen: Standardhygiene statt Isolierung. Chirurg 2009; 80: 49-61

4 Wenzel RP, Bearman G, Edmond MB. Screening for MRSA: a flawed hospital infection control intervention. Infect Control Hosp Epidemiol 2008; 29: $1012-1018$

5 Schulze-Röbbecke R. Isolierung infektiöser Patienten - auf die Übertragungswege kommt es an. Krankenh. hyg up2date 2006; 1: $97-114$ 